\title{
Elimination of lymphatic filariasis in South East Asia
}

\author{
Expanding treatment options alongside ensuring high coverage of mass drug administration can \\ accelerate progress in elimination of lymphatic filarisis, say Sabine Specht and colleagues
}

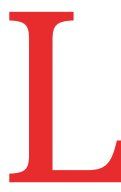

ymphatic filariasis is a tropical disease that affects about 70 million people worldwide. ${ }^{1}$ It is caused by infection with the parasitic nematodes Wuchereria bancrofti, Brugia malayi, or Brugia timori and is transmitted through mosquitoes. Chronic infection causes lymphatic dysfunction, resulting in progressive, irreversible swelling of the limbs and genitals (box 1). Filarial induced lymphoedema is the second leading cause of disability in the world, accounting for about two million disability adjusted life years lost. ${ }^{1}$ The associated social stigma often causes mental health problems and poverty because of loss of employment. ${ }^{1}$

The third sustainable development goal calls for elimination of neglected tropical diseases, including filariasis, by 2020. Sixty three per cent of the population at risk of lymphatic filariasis and $50 \%$ of the people infected worldwide live in South East Asia. India alone harbours $40 \%$ of the world's burden of disease. ${ }^{2}$ The region has made considerable progress towards elimination, yet several challenges remain. We present an overview of the global efforts to eliminate filariasis and progress made in South East Asia, and discuss key priorities.

\section{Global elimination efforts}

The World Health Organization launched the global programme to eliminate lymphatic filariasis in 2000. This programme

\section{KEY MESSAGES}

- Mass administration of microfilaricidal drugs has reduced new infections of filariasis

- Current challenges include management of patients with chronic manifestations, such as lymphoedema and hydrocele, and the uneven prevalence, with persisting transmission hotspots

- New drugs and regimens that kill adult worms (eg, triple therapy) and alleviate lymphoedema can help accelerate elimination efforts comprises two key strategies: mass drug administration to prevent infection, and management of morbidity and prevention of disability.

\section{Mass drug administration}

Mass drug administration entails annual distribution of diethylcarbamazine in combination with albendazole for a minimum of five years in an endemic area. ${ }^{3}$ These drugs are mainly microfilaricidal. The goal is to achieve a coverage of more than $65 \%$ of the population. It is based on the premise that repeated mass drug administration will reduce the microfilaria density in the community and thus halt transmission and new infections. Up to 2015, the programme has provided more than 6.7 billion treatments to over 850 million people at least once in 66 countries. Mass administration is estimated to have cured or prevented up to 96 million new cases of lymphatic filariasis and averted more than $\$ 100 \mathrm{bn}$ of lifetime economic loss. ${ }^{4}$ Since 2000 , the number of cases of filarial induced hydrocele has declined by about $49 \%$ to 19.4 million, and the number of cases of filarial induced lymphoedema by $23 \%$ to 16.7 million. ${ }^{4}$

\section{Managing chronic disease}

Long term care is important to prevent and treat chronic manifestations of filariasis. Treatment for lymphoedema includes good hygiene (regular washing with soap and water; skin and nail care), use of topical antibiotics or antifungal agents, exer- cise, and appropriate footwear. Providing a basic package of care to manage morbidity has been shown to reduce the frequency of acute attacks of adenolymphangitis that drive the progression of lymphoedema. ${ }^{5}$

Microfilaricidal drugs have little benefit in infected individuals with lymphoedema and hydrocele. ${ }^{7}$ A recent trial involving 105 children with filariasis in India showed a possible benefit in reversing lymph dilation early in the course of disease, ${ }^{8}$ and a few observational reports have also noted a benefit. ${ }^{910}$ Further evidence is needed on their role in preventing the development of lymphoedema and associated disfigurement.

\section{Monitoring impact}

The number of people requiring mass drug administration fell from 1.41 billion in 2011 to 856 million in 2016 . $^{1}$ It is expected that mass administration will no longer be required when the prevalence of infection has been reduced to low levels, such as microfilariae in $<1 \%$ of the population or antigenaemia in $<2 \%$ of the population. ${ }^{11}$

After five effective rounds of mass drug administration, a school based transmission assessment survey is conducted. Antigen levels are recorded in 6-7 year old children in the endemic area using a filariasis test strip. If the levels meet cut-off criteria suggesting transmission has been arrested, mass drug administration can be stopped and surveillance used

Box 1: Course of lymphatic filariasis

- Adult filarial parasites reside in the lymphatic vessels of an infected person for up to eight years and produce thousands of first stage larvae (microfilaria)

- Mosquitoes of the genera Aedes, Anopheles, Culex, and Mansonia ingest microfilaria during blood meals from humans and these develop into an infective larval stage

- Larvae enter humans through the wound made by a mosquito, where they migrate and settle in the lymphatics to mature into adult worms and complete the cycle

- Lymphatic dysfunction in response to the parasites provokes severe morbidity, including progressive, irreversible swelling of the limbs (elephantiasis) and genitals (hydrocele) with acute adenolymphangitis or acute secondary bacterial infection

- Infection often occurs early in childhood in endemic areas, but clinical signs appear much later. Once triggered, symptoms may progress even after the parasites have died, being sustained by opportunistic bacterial and fungal infections 
instead. Transmission surveys are repeated after one and two years. If these are successful, the region can be validated for certification of elimination. If transmission is still ongoing on assessment, mass drug administration has to be continued.

\section{Progress in South East Asia}

South East Asian countries are at different stages of implementation of the global elimination programme (table 1). In 2016, the region achieved mass drug coverage of $60.7 \%$ of the population in endemic areas. ${ }^{1}$ Sri Lanka, Thailand, and Maldives have achieved the criteria for elimination of lymphatic filariasis. Bangladesh has stopped mass drug administration and is presently under surveillance. ${ }^{1}$

A guiding example in the region is Sri Lanka's Anti Filariasis Campaign, in which three rounds of diethylcarbamazine were followed by five annual rounds of diethylcarbamazine in combination with albendazole distributed in all eight endemic districts between 2002 and 2006. Two post-drug administration surveillance assessments were conducted in 2011. $13^{12}$ and repeated in areas with continued transmission in 2016. All but three areas showed strongly reduced disease transmission, and it is expected that the incidence will fall to zero without further mass drug administration. ${ }^{13}$

\section{Remaining challenges}

Some countries that have completed five annual rounds of mass drug administration are now struggling with suboptimal results on the transmission assessment survey. Elimination efforts have proved challenging in larger countries such as India, with 256 districts involved. Full implementation has not been achieved, and continued transmission is noted in surveys. As such, elimination may not be feasible by 2020 using currently available tools. ${ }^{14}$

Efforts to maintain high mass drug administration coverage must continue. Factors that can interfere with maintaining sufficiently high coverage include insufficient political will, inadequate health infrastructure, logistical issues, systematic non-compliance, and the risk of drug resistance. Recrudescence of infection owing to migration of infected people into areas with interrupted transmission presents a major challenge to elimination efforts.

\section{Next steps}

\section{Surveillance}

The Sri Lanka experience shows the importance of robust surveillance after mass drug administration to identifiy remaining transmission hot spots. The spatial distribution of lymphatic filariasis where one community may be non-endemic but a neighbouring village has a 30\% prevalence makes it particularly difficult to obtain representative data, and success rates may be overinterpreted. In the past, prevalence of lymphatic filariasis was longitudinaly captured in large geographical areas to reduce surveillance costs. Smaller units compensate for spatial prevalence and are more sensitive for detecting persistence or resurgence of lymphatic filariasis.

Using a point-of-care antibody test in combination with xenomonitoring (the detection of parasites in mosquitoes) has been shown to be more sensitive than the antigen testing currently used for detecting low level transmission. ${ }^{12} 13$ Such focused elimination strategies are costly, however, and must be weighed against the costs of upscaling or re-starting mass drug administration if transmission persists. ${ }^{15}$ A tipping point may be reached, at least in some areas, where test and treat (that is, treatment only of those diagnosed as infected) is likely to become more cost effective, even if it requires 5-10 day treatment instead of a single dose yearly for 5-10 years.

\section{Morbidity management}

Morbidity management is even more challenging and must be continued in endemic communities even after mass drug administration has stopped, because affected patients remain in these communities. An accurate assessment of filarial cases has proved difficult. Severe lymphoedema is under-reported in Africa, ${ }^{16}$ while reporting from South East Asia has increased over the past few years. ${ }^{1}$

Health systems should be strengthened to deliver a minimum package of care to all affected individuals, with a goal of achieving complete geographical coverage. WHO has developed a toolkit for managing morbidity and preventing disability for endemic countries that must be integrated into primary healthcare alongside continuing mass drug administration. Training will further support patients to continue care and to improve their quality of life.

\section{New treatment and control options}

Expanding the toolbox to prevent and treat filarial infections will help progress towards elimination. Beneficial effects of bednets have been reported from areas with Anopheles transmission and persistent lymphatic filariasis in Papua New Guinea, where infection is transmitted by indoor biting mosquitoes. The use of impregnated bednets as well as treatment has been suggested for remaining lymphatic filariasis hot spots. ${ }^{1718}$

New drugs enabling reversal of lymphoedema would be highly beneficial. This has also become imperative in view of sustainable development goal 3.8, which targets individual wellbeing and thus calls for individual cure and not merely epidemiological "control as a public health problem."

Since the adult worm confers pathology in lymphatic filariasis, the ultimate goal for a new drug is to kill or sterilise adult worms. A pilot study in 24 patients showed a possible sterilising effect with the addition of ivermectin to the existing treatment regimen (diethylcarbamazine with albendazole). A single dose triple drug therapy (ivermectin in combination with diethylcarbamazine and albendazole) achieved almost total clearance of microfilaraemia at 36 hours. This effect was sustained in all patients at one year (12 patients) and half the patients at two

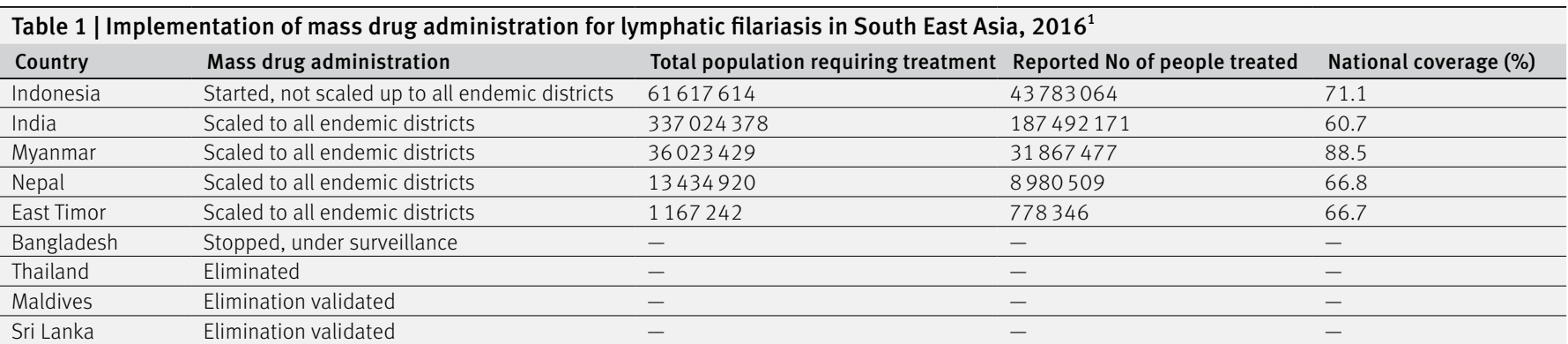


years, compared with the usual two drug regimen, where 11 of 12 patients tested positive for microfilaria at one year. ${ }^{19}$ The triple drug therapy could accelerate interruption of transmission by reducing the number of annual rounds of mass drug administration required to achieve the elimination target. Two to three rounds of treatment with ivermectin in combination with diethylcarbamazine and albendazole may be sufficient to reduce community microfilaraemia to below the threshold level, rather than five to six rounds of dual therapy. ${ }^{2021}$ This would be particularly useful to accelerate progress in countries left behind through delays in mapping or initiation of mass drug administration. In 2017, WHO provisionally approved the use of triple drug therapy to interrupt transmission of lymphatic filariasis infection, and guidelines have been released for its use in Asia, where onchocerciasis and loiasis are not endemic. ${ }^{22}$ India is currently preparing to start the triple drug therapy as part of an accelerated national programme.

Two trials from Africa have shown a positive effect of a six week course of doxycycline in reducing lymphoedema severity in the early stages in patients with filariasis ${ }^{2324}$ beyond that seen with improved hygiene alone. This improvement was independent of current filarial infection. ${ }^{24}$ Currently, five multicentre placebo controlled trials are being conducted (three in Africa and two in Asia) and will provide evidence to determine whether doxycycline can be included as an adjunct therapy for morbidity management. Understanding its effect on the adult worm and microfilaria will help inform its use in reducing transmission as well.

To expand the toolbox for anti-filarial drugs, the Drugs for Neglected Diseases initiative, together with its partners from academia and industry, is developing new anti-wolbachial and direct acting drugs. Two of these, emodepside and ABBV4083, are orginal or modified veterinary drugs and are now in phase I development for use in humans; several others are in the drug pipeline. While these drugs will be developed primarily for use against onchocerciasis, for which apart from doxycycline no safe macrofilaricide exists, their indication may extend to include lymphatic filariasis as well.

However, it has also become clear that higher efficacy drugs do not compensate for low coverage. Decision makers must assess the feasibility of, and rationale for, investing in new strategies for elimination of lymphatic filariasis, taking into consideration the costs of the programme to ensure wide coverage. Successful elimination of lymphatic filariasis will depend on more than monetary investment. Going forward, political will and continued public engagement and community ownership will be critical. ${ }^{25}$

Competing interests: We have read and understood BMJ policy on declaration of interests and have no relevant interests to declare.

Provenance and peer review: Commissioned; externally peer reviewed.

This article is one of series commissioned by The BMJ in collaboration with the Drugs for Neglected Diseases initiative (DNDi). The BMJ retained full editorial control over external peer review, editing, and publication. Open access fees are funded by the DNDi, Geneva.

Sabine Specht, head of filarial clinical programme ${ }^{1}$

T K Suma, professor of internal medicine ${ }^{2}$

Belen Pedrique, researcher ${ }^{1}$

Achim Hoerauf, professor of microbiology

${ }^{1}$ Drugs for Neglected Diseases initiative, Geneva, Switzerland

${ }^{2}$ Filariasis Research Unit, Government T D Medical College, Alappuzha, Kerala, India

${ }^{3}$ Institute for Medical Microbiology, Immunology and Parasitology, University Hospital Bonn, Germany

Correspondence to: SSpecht

sspecht@dndi.org

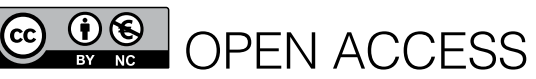

This is an Open Access article distributed in accordance with the Creative Commons Attribution Non Commercial (CC BY-NC 4.0) license, which permits others to distribute, remix, adapt, build upon this work non-commercially, and license their derivative works on different terms, provided the original work is properly cited and the use is non-commercial. See: http://creativecommons.org/ licenses/by-nc/4.0/.

\section{Check for updates}

1 World Health Organization. Summary of global update on preventive chemotherapy implementation in 2016: crossing the billion. Wkly Epidemiol Rec 2017;92:589-93.

2 WHO SEARO. Neglected tropical diseases: Lymphatic filariasis. http://www.searo.who.int/ entity/vector_borne_tropical_diseases/topics/ lymphatic_filariasis/en/

3 World Health Organization. Lymphatic filariasis: progress report 2000-2009 and strategic plan 2010-2020. WHO/HTM/NTD/PCT/20106. 2010.

4 Ramaiah KD, Ottesen EA. Progress and impact of 13 years of the global programme to eliminate lymphatic filariasis on reducing the burden of filarial disease. PLOS Neglected Tropical Diseases 2014;8:e3319. doi:10.1371/journal.pntd.0003319

5 Addiss DG, Louis-Charles J, Roberts J, et al. Feasibility and effectiveness of basic lymphedema management in Leogane, Haiti, an area endemic for bancroftian filariasis. PLOS Neglected Tropical Diseases 2010;4:e668. doi:10.1371/journal. pntd.0000668

6 Kerketta AS, Babu BV, Rath K, Jangid PK, Nayak AN, Kar SK. A randomized clinical trial to compare the efficacy of three treatment regimens along with footcare in the morbidity management of filarial lymphoedema. Trop Med Int Health 2005;10:698-705. doi:10.1111/j.13653156.2005.01442.x

7 World Health Organization. Report on the mid-term assessment of microfilaraemia reduction in sentinel sites of 13 countries of the Global Programme to Eliminate Lymphatic Filariasis. Wkly Epidemiol Rec 2004:79:358-65.

8 Kar SK, Dwibedi B, Das BK, Agrawala BK, Ramachandran CP, Horton J. Lymphatic pathology in asymptomatic and symptomatic children with Wuchereria bancrofti infection in children from Odisha, India and its reversal with DEC and albendazole treatment. PLOS Neglected Tropical Diseases 2017;11:e0005631. doi:10.1371/journal. pntd.0005631

9 Moore TA, Reynolds JC, Kenney RT, Johnston W, Nutman TB. Diethylcarbamazine-induced reversal of early lymphatic dysfunction in a patient with bancroftian filariasis: assessment with use of lymphoscintigraphy. Clin Infect Dis 1996;23:100711. doi:10.1093/clinids/23.5.1007

10 Shenoy RK. Clinical and pathological aspects of filarial lymphedema and its management. Korean | Parasitol 2008;46:119-25. doi:10.3347/ kjp.2008.46.3.119

11 World Health Organization. Monitoring and epidemiological assessment of mass drug administration in the global programme to eliminate lymphatic filariasis: a manual for national elimination programmes. WHO, 2011. https://www.who.int/iris/ handle/10665/44580.

12 Rao RU, Nagodavithana KC, Samarasekera SD, et al. A comprehensive assessment of lymphatic filariasis in Sri Lanka six years after cessation of mass drug administration. PLOS Neglected Tropical Diseases 2014;8:e3281. doi:10.1371/journal. pntd.0003281

13 Rao RU, Samarasekera SD, Nagodavithana KC, et al. Reassessment of areas with persistent lymphatic filariasis nine years after cessation of mass drug administration in Sri Lanka. PLOS Neglected Tropical Diseases 2017;11:e0006066. doi:10.1371/journal. pntd.0006066

14 Krentel A, Fischer PU, Weil GJ. A review of factors that influence individual compliance with mass drug administration for elimination of lymphatic filariasis. PLOS Neglected Tropical Diseases 2013;7:e2447. doi:10.1371/journal.pntd.0002447

15 Gedge LM, Bettis AA, Bradley MH, Hollingsworth TD, Turner HC. Economic evaluations of lymphatic filariasis interventions: a systematic review and research needs. Parasites and Vectors 2018;11:75. doi:10.1186/s13071-018-2616-z

16 Stanton MC, Mkwanda SZ, Debrah AY, et al. Developing a community-led SMS reporting tool for the rapid assessment of lymphatic filariasis morbidity burden: case studies from Malawi and Ghana. BMC Infectious Diseases 2015;15:214. doi:10.1186/ s12879-015-0946-4

17 Reimer LJ, Thomsen EK, Tisch DJ, et al. Insecticidal bed nets and filariasis transmission in Papua New Guinea. N Engl J Med 2013;369:745-53. doi:10.1056/NEJMoa1207594

18 Eigege A, Kal A, Miri E, et al. Long-lasting insecticidal nets are synergistic with mass drug administration for interruption of lymphatic filariasis transmission in Nigeria. PLOS Neglected Tropical Diseases 2013;7:e2508. doi:10.1371/journal. pntd.0002508

19 Thomsen EK, Sanuku N, Baea M, et al. Efficacy, safety, and pharmacokinetics of coadministered diethylcarbamazine, albendazole, and ivermectin for treatment of Bancroftian filariasis. Clin Infect Dis 2016:62:334-41. doi:10.1093/cid/civ882

20 Stolk WA, Prada JM, Smith ME, et al. Are alternative strategies required to accelerate the global elimination of lymphatic filariasis? Insights from mathematical models. Clin Infect 
Dis 2018;66(suppl_4):S260-6. doi:10.1093/cid/ ciy003

21 Irvine MA, Stolk WA, Smith ME, et al. Effectiveness of a triple-drug regimen for global elimination of lymphatic filariasis: a modelling study. Lancet Infect Dis 2017;17:451-8. doi:10.1016/S14733099(16)30467-4

22 World Health Organization. Guideline-Alternative mass drug administration regimens to eliminate lymphatic filariasis. WHO/HTM/NTD/PCT/201707. 2017.

23 Debrah AY, Mand S, Specht S, et al. Doxycycline reduces plasma VEGF-C/SVEGFR-3 and improves pathology in lymphatic filariasis. PLoS Pathogens 2006;2:e92. doi:10.1371/journal. ppat.0020092

24 Mand S, Debrah AY, Klarmann U, et al. Doxycycline improves filarial lymphedema independent of active filarial infection: a randomized controlled trial. Clin Infect Dis 2012;55:621-30. doi:10.1093/cid/cis486 25 Damian GW, Rabinovich R. Guidelines for preparing an eradication investment case. In: Cochi SI, Dowdle WR, eds. Disease eradication in the 21st century: Implications for global health. MIT Press, 2011: 149-56

Cite this as: BMJ 2019;364:k5198 http://dx.doi.org/10.1136/bmj.k5198 